\title{
Interkulturelle Kompetenz in der Arbeit mit traumatisierten Menschen mit Fluchterfahrung
}

\author{
Christine Pichlhöfer
}

Online publiziert: 30 . September 2021

(C) Der/die Autor(en) 2021

Zusammenfassung Im Artikel der Zeitschrift für Psychodrama und Soziometrie wird die psychotherapeutische Arbeit mit traumatisierten Menschen mit Fluchterfahrung, unter anderem auch anhand von Fallvignetten, besprochen. Es geht um die Begegnung mit Menschen, die ihr Leben bisher in einem anderen Land verbracht haben und sich aufgrund zerbrochener Sicherheiten dazu gezwungen sahen, dieses $\mathrm{zu}$ verlassen. Flucht ist nie freiwillig. Wie viel muss ich über eine andere Kultur wissen und wie kann ich mich gleichzeitig vor verallgemeinernden Vorannahmen bewahren? Oft ist es ein therapeutisches Arbeiten mit traumatisierten Menschen mit ungeklärtem Aufenthaltsstatus und damit auch mit fehlender Sicherheit. Die Suche nach Ressourcen im Inneren ist ein intensiver Prozess, jene im Außen eine Herausforderung. Begegnung auf Augenhöhe und der gemeinsame Prozess des Findens eines passenden therapeutischen Prozesses erfordern ein besonderes Einlassen in uns oft unbekannte Welten.

Schlüsselwörter Interkulturalität · Transkulturalität · Flucht · Trauma · Psychodrama $\cdot$ Flüchtlinge $\cdot$ Spannung $\cdot$ Rollenerweiterung $\cdot$ Diversität $\cdot$ Inklusion

Christine Pichlhöfer, DSAin MSc

Praxisgemeinschaft, Marokkanergasse 19/3, 1030 Wien, Österreich

Donau-Universität Krems, Krems, Österreich

E-Mail: psychodrama@pichlhoefer.eu 


\title{
Intercultural competence in working with traumatized people with experience of being on the run
}

\begin{abstract}
The article in the journal Zeitschrift für Psychodrama und Soziometrie discusses psychotherapeutic work with traumatized people with experience of being on the run, including case vignettes. It is about the encounter with people who have spent their lives in another country and were forced to leave it because of broken securities in this country. Escape is never voluntary. How much do I need to know about another culture and how can I simultaneously protect myself from generalizing assumptions? Often it is a therapeutic work with traumatized people with an unresolved residence status and thus a lack of security. The search for resources inside is an intensive process, those outside are a challenge. Encounter on a par and the common process of finding a suitable therapeutic process requires a special engagement in often unknown worlds.
\end{abstract}

Keywords Interculturality $\cdot$ Transculturality $\cdot$ Escape $\cdot$ Trauma $\cdot$ Psychodrama Refugees $\cdot$ Tension $\cdot$ Role extension $\cdot$ Diversity $\cdot$ Inclusion

\section{Einleitung}

Menschen mit Fluchterfahrung haben nicht selten eine Reihe von traumatisierenden Erfahrungen erlebt, bis sie im Aufnahmeland ankommen. Der schwerwiegenden Entscheidung zu fliehen liegen extreme Erfahrungen im Herkunftsland zugrunde: Krieg, Verfolgung, Vertreibung, Folter, Diskriminierung, Misshandlung, Vergewaltigung, Mord an nahestehenden Menschen, Morddrohungen etc. Dazu kommen Belastungen auf den oft langen Fluchtwegen mit wenig Schlaf und neuerlichen Gewaltoder Missbrauchserfahrungen, das Sterben und Begraben von Mitflüchtenden etc. Auch die Trennung von FreundInnen und Familie, Hunger, Kälte, Angst vor dem Entdecktwerden, neuerliche Drohungen, Ablehnung etc. führen zu einem Zustand ständiger innerer Anspannung, wodurch es auch zu weiteren Traumata kommen kann. „Körper und Seele finden sich in einem Überlebensmodus, der das Leid und die Not aushalten lässt.“ (Imm-Bazlen und Schmieg 2017, S. 11).

Endlich in einem „sicheren Land“ angekommen, besteht die Gefahr weiterer Belastungen durch die Erfahrung von Ablehnung und Ausgrenzung und der Angst bei laufenden oder abgelehnten Asylverfahren ins Herkunftsland zurückgeschoben zu werden. Erstaunlicherweise gibt es trotz extremer Erfahrungen Menschen mit Fluchterfahrung, die nicht traumatisiert sind und es gibt Menschen, die ihre traumatischen Erfahrungen soweit integrieren und verarbeiten können, dass sie keiner weiteren Behandlung bedürfen. Menschen mit Fluchterfahrung werden nicht selten auf diese eine Rolle - Flüchtling - reduziert. Die Philosophie des Psychodramas erkennt die Rollenvielfalt im Menschen und schützt vor einseitiger Sichtweise und der Gefahr, als TherapeutIn das Gegenüber lediglich auf eine Rolle zu reduzieren. In der Psychodrama-Psychotherapie findet ein Wahrnehmen, Wiederentdecken und Weiterentwickeln von Rollen statt, die weit über jene der/des Geflüchteten hinausgehen. Dies ist - verbunden mit interkultureller Reflexion und Haltung auf beiden 
Seiten - ein wichtiger Teil im Prozess der Traumabearbeitung und auf dem Weg zur Inklusion. „Moreno glaubt, dass jeder Mensch seinen ihm eigenen Weg finden muss und finden kann.“ (Trinkel und Kern 2018, S. 1).

\section{Asyl und Flucht}

Ein grundlegendes Wissen über folgende Begriffe, die im Zusammenhang mit dem Aufenthaltsstatus von Flüchtlingen stehen, kann beim Perspektivenwechsel und im Prozess allgemein hilfreich sein (vgl. dazu UNHCR 2016, S. 6):

- Asylsuchende warten darauf, wie das Asylverfahren ausgeht. Sie haben um Schutz vor Verfolgung angesucht. Die zuständige Behörde ist dabei zu prüfen, ob einem Menschen Schutz gewährt oder der Antrag abgelehnt wird. Diese Phase ist zusätzlich zu erlebten traumatischen Erfahrungen, eine schwere psychische Belastung.

- Subsidiär Schutzberechtigte bekommen einen zeitlich begrenzten Schutz, weil z. B. im Herkunftsland Krieg herrscht. Sie müssen den subsidiären Schutz immer wieder verlängern lassen. Sie haben Zugang zum Arbeitsmarkt und zu Sozialleistungen (je nach Bundesland unterschiedlich).

- Ein anerkannter Flüchtling nach der Genfer Flüchtlingskonvention ist ,eine Person, die ihr Herkunftsland verlassen musste, weil sie aufgrund ihrer Religion, Nationalität, Rasse, politischen Meinung oder Zugehörigkeit zu einer bestimmten sozialen Gruppe (darunter fallen z. B. Homosexuelle) verfolgt wird oder begründete Furcht vor Verfolgung hat." (UNHCR 2016, S. 6). Das Recht auf Asyl ist ein Menschenrecht. Anerkannte Flüchtlinge haben Zugang zum Arbeitsmarkt und Anspruch auf Sozialleistungen, z. B. Krankenversicherung. Aber auch sie dürfen nicht mehr in ihr Heimatland zurück. Nach der Erleichterung dem Schrecken entkommen und an einem sicheren Ort zu sein, entsteht nicht selten nach einigen Jahren große Sehnsucht nach dem Herkunftsland, danach, geliebte Menschen und Orte der Kindheit wiederzusehen. Das ist allerdings nicht möglich.

\section{Trauma}

„Trauma ist immer eine bis ins Körperliche durchgreifende Erfahrung von extremer Hilflosigkeit und Verlust, bei welcher die bisher gelernten Abwehr- und Problemlösungsstrategien eines Individuums auf breiter Front versagen." (Ottomeyer 2004, S. 348). Das eigene Wertesystem bricht zusammen, das Vertrauen in sich selbst, in andere Menschen, in die ganze Welt ist schwer erschüttert. Der Glauben an Transzendentes, der zuvor womöglich als Ressource gedient hat, geht verloren. Im ICD10 wird beschrieben, dass ein solches Ereignis bei fast jedem Menschen ,eine tiefe Verzweiflung hervorrufen würde" (Dilling und Freyberger 2016, S. 173ff.), wenn die außergewöhnliche Bedrohung die eigenen Bewältigungsmöglichkeiten übersteigt und ein Mensch ihr schutzlos ausgeliefert ist. Gelingt eine Aufarbeitung nicht, so kann es zu einer psychischen Störung kommen. Menschen mit Fluchterfahrung haben eine erhöhte Vulnerabilität für die Entwicklung von psychischen Störungen, v. a. für eine posttraumatische Belastungsstörung (kurz: PTBS, F43.1 nach ICD-10) (vgl. 
Schnabel und Gottwald 2017, S. 73). Oft handelt es sich um komplexe Traumatisierungen, die mit mehreren oder sich wiederholenden außergewöhnlich belastenden Ereignissen zu traumatisierendem Erleben führen. Wird ein Psychotrauma nicht bewältigt, so kann es zu weiteren Krankheiten führen. Wintersberger nennt die PTBS im Zusammenhang mit folgenden komorbiden Störungen: Depressionen, somatoforme Störungen, Angststörungen, Sucht, dissoziative Symptome (Wintersberger 2020, S. 56). „Trauma ist immer ein ,Zerbrechen der Erzählung“. [...] Die Erzählung ist immer fragmentiert, mit Rissen im roten Faden." (Ottomeyer 2004, S. 350). Nicht der Mensch ist verrückt oder unnormal, wie er auf eine traumatische Situation reagiert (hat), sondern die Situation war unnormal und verrückt. Reaktionen haben immer einen Sinn in der Situation, sie haben geholfen zu überleben. Hier besitzt das Psychodrama „mit zentralen Paradigmen und den daraus resultierenden Haltungen beste Voraussetzungen für die Arbeit mit Traumafolgestörungen." (Pruckner 2018, S. 376). Wenn sowohl Kampf- als auch Fluchtreflexe versagen, kann es zu einem Erstarrungsreflex kommen. Hilflosigkeit und Ohnmacht entstehen, wie bei Achmed ${ }^{1}$ im nachfolgenden Beispiel: „In dieser traumatischen ,Zwangssituation“ von „,no Fight - no Flight - Freeze“" setzen die archaischen autoprotektiven Notfallreaktionen ein: Dissoziation (Erstarren, Wahrnehmungsverzerrung und -ausblendung); in der Übererregung: Submission (Unterwerfung) entspricht Dissoziation in der Übererregung (,Totstellreflex“).“ (Hüther et al. 2010, S. 21). Die Reaktion ist einer Panikattacke mit Herzrasen und Todesangst ähnlich, es kommt zur Dissoziation, um sich der massiven Reizüberflutung zu entziehen.

Achmed war es sehr wichtig zu wissen, dass er nicht ,im Kopf kaputt ist““. Er war voller Angst, nicht nur um seine Geschwister, die im Herkunftsland verblieben waren, ohne Kontaktmöglichkeit zu ihnen, sondern auch um die Gefahr abgeschoben zu werden und vor dem Entdecktwerden durch seine Folterer, die ihn auch hier finden könnten. Und nun kam auch noch die Angst dazu, verrückt zu sein oder zu werden, nachdem er bei einer Fahrscheinkontrolle im Bus erstarrt war. Er saß auf einem Sitz und wurde von zwei männlichen Kontrolleuren nach seinem Fahrschein gefragt. Er hatte einen bei sich. Er weiß nicht genau, was passiert ist. Er konnte weder sprechen, noch sich bewegen - und somit auch seinen Fahrschein nicht vorweisen. Er wurde aufgefordert auszusteigen, seine Muskeln versagten. Die beiden Kontrolleure packten ihn jeweils links und rechts, um ihn aus dem Bus zu bringen. Draußen am Gehsteig ließen sie ihn los, er sackte auf dem Boden zusammen. Ab jetzt wollte er nicht mehr Bus fahren.

Die psychotherapeutische Behandlung von Achmeds Traumafolgestörung fand über einen langen Zeitraum statt. Ich habe anschauliche Interventionen ausgewählt und in der Darstellung komprimiert. Der Therapieprozess geht immer von

\footnotetext{
${ }^{1}$ Die Namen und Situationen aller Menschen mit Fluchterfahrung wurden von mir im ganzen Artikel (nochmals) geändert. Die genauen Fallbeschreibungen wurden bewusst verzerrt, nicht nur um die Privatsphäre zu wahren, sondern v. a. auch um bedrohte Menschen keiner neuerlichen Gefahr auszusetzen und um sie zu schützen. Ihre Namen und u. U. auch andere Angaben mussten sie fallweise selbst schon lange vorher ändern, zum Schutz vor Verfolgung, Folter, Erpressung (,,Deine Familie, Deine FreundInnen ... wir wissen, wo wir sie finden ...!" ).
} 
Stabilisierung und Ressourcenarbeit aus, davon die Selbstwirksamkeit zu erhöhen - vorerst bei positiven Gefühlen, um zu einem späteren Zeitpunkt ein größeres Gefühlsspektrum erlebbar zu machen und mehr Einfluss auf das eigene Handeln zu gewinnen. „Es ist wichtig, das Bedürfnis der PatientInnen nach Kontrolle ebenso wie alle anderen Bewältigungsstrategien der PatientInnen zu würdigen.“ (Reddemann 2019, S. 25). Eine vorsichtige, kontrollierte, sanfte Annäherung an das Trauma kann erst stattfinden, wenn sich der/die KlientIn in Sicherheit befindet, wenn der traumatisierte Mensch Stabilisierungsmöglichkeiten für sich erarbeitet hat und das Vertrauen in die psychotherapeutische Beziehung hält. Auch mit Psychoedukation ist behutsam umzugehen, da immer wieder Intrusionen (Wiedererleben in Form von Flashbacks, Nachhallerlebnisse) unkontrolliert auftauchen können - mit allen bekannten Folgen, bis zu heftigen körperlichen Reaktionen, so als wäre die Person wieder in der traumatischen Situation, ohne neue Handlungsmöglichkeiten (vgl. Reddemann und Dehner-Rau 2018, S. 56). Bei Achmed bedeutet das, mit ihm gemeinsam auf lange Sicht über menschliche Überlebenssysteme zu sprechen und über die Verbindung des Erstarrens (freeze) bei der Fahrscheinkontrolle mit der Erfahrung, die er im lebensbedrohlichen Kriegsgeschehen gemacht hat. Für eine sehr vorsichtige, kontrolliert gesteuerte sanfte Annährung an das Trauma braucht es noch Stabilisierung. Treten bei einer posttraumatischen Belastungsstörung Symptome wie Übererregung, Schwindel, Flashbacks und psychosomatische Beschwerden auf, so gilt es immer zuerst psychotherapeutische Krisenmaßnahmen wie Atemübungen oder kognitive Stimulation einzuleiten, um einen völligen Zusammenbruch zu verhindern (vgl. Ottomeyer 2002, S. 154). Für die nächste Zeit allerdings liegt der Fokus in der Zurückgewinnung der Handlungsmacht beim Busfahren, das ist unmissverständlich Achmeds Wunsch:

Mit einer Psychodrama-Distanzierungs-Technik, deren Grundlagen auch in der Psychotraumatologie zu finden sind, nähern wir uns dem Thema der beunruhigenden vorübergehenden Handlungsunfähigkeit, die Achmed bei der Fahrscheinkontrolle erstarren ließ: Ich begleite Achmed beim Kreieren einer Szene, in der wir vor der weißen Wand im Kino stehen (Achmed möchte lieber nicht sitzen) und uns gemeinsam folgenden Film ansehen, die Fernbedienung (ein Stück Holz) in Achmeds Hand: Ein Mann, Achmed nennt ihn Mohammed, wird von zwei Kontrolleuren im Zug nach seinem Fahrschein gefragt. Wir gehen die Bilder durch, Achmed stoppt immer wieder mit seiner Fernbedienung und wir reden über Mohammed und sein Handeln, die Uniformierten, das Sprechen, den Zug. Ich bestätige und ergänze, v. a. hebe ich hervor, dass Mohammed nichts Schlimmes gemacht hat, dass es aber in diesem Moment für ihn nicht möglich war zu sprechen oder sich zu bewegen (Schutz vor Selbstabwertung). Das ist okay, das kann in Zukunft anders werden (Entwicklung ist möglich). Er hat getan, was möglich war (Anerkennen, Bestätigung). Ein Zwiegespräch zwischen Achmed und mir als Beobachtende von Mohammeds Handlungen im Zug bringt Ruhe und mehr Sicherheit für Achmed.

Durch die Distanzierung in der Beobachterrolle wird es für Achmed möglich, wieder Kontrolle über seine Gefühle zu erlangen - dies trägt zur Stabilisierung bei und wirkt der Angst entgegen. In Achmed wächst das Vertrauen in 
sich, eine ähnliche Situation in Zukunft bewältigen zu können. Er entwirft eine Zukunftsszene für die nächste Kontrolle: Und zwar wird er nicht sprechen, sondern nur den Fahrschein zeigen, diese neu entwickelte Möglichkeit entlastet ihn. Stabilisierung und Ressourcenarbeit stehen noch lange im Vordergrund.

\section{Interkulturelle/transkulturelle Kompetenz}

Es ist wichtig, sich bewusst zu machen, welcher Kulturbegriff der interkulturellen bzw. transkulturellen Kompetenz zugrunde liegt und was mit Kultur verbunden wird, z. B. Ethnie, Nationalität, Religion, Sprache, Tradition. Des Weiteren ist zu reflektieren, welche Konzepte wir dazu erlernt haben und wie sie unser Handeln beeinflussen. Interkulturelle Psychotherapie verlangt von TherapeutInnen eine selbstreflexive und diversitätssensible, wertungsabstinente Haltung, die einerseits durch eine offen-neutrale sowie gleichzeitig parteiliche Position geprägt ist (vgl. Schlichtmeier 2018). Es ist eine prozesshafte Kompetenz, die auch Wissen über Diskriminierung oder subjektive Bedeutung von Religion beinhaltet. Begreifen wir Kulturen als hybride und fluide Gebilde, so gelangen wir zur Transkulturalität. „Während der Begriff Interkulturalität [...] ein Zwischen den Kulturen suggeriert, bezieht sich Transkulturalität auf etwas Übergreifendes und die National- bzw. Einzelkulturen Transzendierendes. [...] Wobei die bestehenden Herrschaftsverhältnisse und Absolutheitsansprüche von dominanten Kulturen und nationalistischen Diskursen hierdurch hinterfragt werden sollen." (Pries und Maletzky 2018, S. 55). Konzentrieren wir uns v.a. bei geflüchteten Menschen auf Kulturdifferenzen, kann es passieren, dass wir vielfältige Phänomene von Diversität innerhalb dieser Kulturen ausblenden. Wir bewegen uns im Therapieprozess in einem Spannungsfeld, das es nicht aufzulösen, sondern mitzutragen und zu halten gilt. Wir eignen uns Wissen und Hintergrundinformationen über eine bestimmte Kultur, ein bestimmtes Land oder eine Religion an. Dieses Wissen ist uns in der Begegnung mit Menschen mit Fluchterfahrung Hilfe und Barriere zugleich. So kann es z.B. von Vorteil sein zu wissen, dass in einem Land Paschtu gesprochen wird, da es Wertschätzung deutlich macht und zeigt, dass man sich damit beschäftigt hat. Andererseits entstehen im gemeinsamen Teleprozess mit den KlientInnen neue Szenen; womöglich gilt es, angeeignetes Wissen wieder zu verwerfen, damit sich ein neues Bild eines Menschen entwickeln kann. Es bleibt ein Prozess, auf den wir uns einlassen.

Zur besseren Nachvollziehbarkeit möchte ich hier ein Beispiel anführen, das in Abwandlungen häufiger vorkommt. Ich habe es selbst miterlebt, aus unterschiedlichen Perspektiven erzählt bekommen und vor kurzem sah ich es zufällig bei einer der Mehrheitsgesellschaft zugehörigen Kabarettistin nicht besonders wertschätzend dargestellt ohne Selbstreflexion, als Schenkelklopfer auf Kosten der vulnerablen Minderheit verarbeitet:

Es ist ein lauer Vor-Corona-Abend in der Ausgehmeile am Gürtel in Wien. Ein etwa 30-jähriger Mann, Danyal, stellt sich vor einem Lokal zu einer Gruppe Menschen, Männer und Frauen, ebenfalls um die 30, alle mit ähnlichem Kleidungsstil, lockere Gespräche, gegenseitiges Kennenlernen. Danyal fragt 
einen der anwesenden, rauchenden Männer nach Feuer für seine Zigarette. Ein freundlicher Mann, Markus, gibt ihm sogleich mit einem Lächeln Feuer und fragt ihn, woher er denn kommt? Die Stimmung von Danyal kippt, er ist empört über die Frage und verlässt schimpfend die Gruppe. Markus und die anderen bleiben etwas durcheinander zurück. Vor allem Markus ist gekränkt; er wollte mit Danyal ein unverfängliches Gespräch beginnen und ihn so auch in die Gruppe einbinden. Er hat sich bemüht und versteht nicht, was passiert ist.

Machen wir nun einen Perspektivenwechsel und stellen uns an die Seite von Danyal: Er kleidet sich wie alle, die dieses Lokal besuchen, hat etwas dunklere Hautfarbe und spricht gebrochen Deutsch. Er wird sehr häufig auf sein Anderssein reduziert. Auch hier wurde er in der Rolle des Anderen, „nicht-von-hier“, adressiert. Das aktiviert aufgrund von häufigen Diskriminierungserfahrungen in der Vergangenheit wiederum seine Rollenkonserve des Gekränkten. Reduzierung auf die Rolle des Anderen bedeutet für ihn in diesem Moment Ablehnung und führt zur Reaktion: „Ich lehne dich ab, weil du mich ablehnst.“

Wie kann eine Situation dieser Art aufgelöst werden? Bei Angehörigen der kulturellen Mehrheit, mit allen Privilegien von in Österreich geborenen und aufgewachsenen Mehrheiten, wozu auch ich mich zähle, braucht es einen Perspektivenwechsel, eine Sensibilisierung, um die Reaktion von Danyal nachvollziehbarer zu machen. „Wahrnehmungen und Zuschreibungen schaffen Fakten. [...] Es geht darum, uns selbst und unser Gegenüber wahrzunehmen als Mensch mit vielschichtigen Identitäten“ (Dommel 2018, S. 467), von denen die Geburt in einem anderen Land oder die Fluchterfahrung nur eine von vielen ist. Es geht darum, Uneindeutiges auszuhalten. Damit sich Danyal in Zukunft besser schützen kann, braucht es einen Entwicklungsprozess hin zur Rollenerweiterung, damit er in solchen Situationen mehr Handlungsmöglichkeiten zur Verfügung hat. Hat Danyal auch noch traumatische Erfahrungen gemacht, so kann ihn eine solche, nach außen harmlos scheinende Szene, in eine schwer zu regulierende Spannungssituation bringen.

Auch in den sozialen Netzwerken können sich menschenverachtende Echokammern (echo chambers) bilden (vgl. dazu Müller und Schwarz 2020). Auf die Frage „Warum bzw. wo ging uns das emanzipatorische Potenzial der digitalen Netzwerke verloren?“ (Judmaier und Pichlhöfer 2016, S. 8) reagiert der in Wien lebende deutsche Management-Student Dominik Lucha und teilt in seinem Instagram-Profil @ wasihrnichtseht Aussagen, die an Menschen mit anderer Hautfarbe gerichtet sind. Er zeigt, wie tief diese verletzen können und wie diskriminierend sie sind. Alltagsrassismus ist tief in der Gesellschaft verankert und selbst wenn ein Mensch anderer Hautfarbe in Österreich geboren ist, wird er oder sie rasch gefragt - und das immer und immer wieder - woher sie/er kommt. Das führt dazu, dass eine Identifikation mit dem eigenen Land erschwert wird und ein Sich-fremd-Fühlen mit sich bringt (vgl. dazu Binder 2020, S. 4). 


\section{Kultur und Hintergrund}

Wie wir sozialisiert werden, macht einen Unterschied, auch wenn ich gerne möchte, dass es keinen macht. Es gilt, sich kulturelle Kompetenz prozesshaft anzueignen, dieser Prozess findet laufend statt. In der Begegnung mit Ashira konnte auch ich mich wieder ein Stück in diesem Prozess weiterentwickeln:

Die 17-jährige Wienerin Ashira erzählt mir von Konzentrationsschwierigkeiten in letzter Zeit in der Schule und von Schlafschwierigkeiten. Ich frage nach dem Einschlafrhythmus, nach Ritualen vor dem Schlafengehen, nach Lebensänderungen und werde kaum fündig, was diese Änderung bewirkt haben könnte. So isst die Familie derzeit zwar relativ spät zu Abend, erst gegen 21 Uhr, aber Ashira geht - so wie meist - gegen 23 Uhr zu Bett.

Selbst aufgewachsen in Österreich mit christlichen Ritualen, denke ich vorerst nicht an einen wichtigen Punkt und auch Ashira hat ihn nicht explizit erwähnt. Es ist gerade Ramadan. Muslimische Familien gestalten diese Fastenzeit sehr unterschiedlich. Ich weiß, dass bei einigen zwischen Sonnenauf- und -untergang weder getrunken, noch gegessen wird, so manch eine/r verzichtet auch auf Nikotin und Alkohol in dieser Zeit, so diesen Genussmitteln nicht ohnehin ganz entsagt wird. Doch was dies im Speziellen bedeutet, weiß ich nur vage. Auch hier gilt wieder: Gut, dass ich jetzt daran denke, dass Ramadan ist, das wird von Ashira auch sehr geschätzt. Gut, dass ich Grundlegendes dazu weiß. Doch gleichzeitig ist es absolut notwendig, dafür offen zu sein, dass es bei Ashira wieder ganz anders sein kann.

Auch in Familien mit christlichen Traditionen kann die Fastenzeit sehr unterschiedlich ausfallen: Die einen verzichten ohnehin das ganze Jahr auf Fleisch, für die anderen wird das zur Besonderheit während der Fastenzeit. Die einen verzichten auf Zigaretten, Alkohol, Sex, Süßigkeiten, Zocken oder schränken ihren Handykonsum ein, die anderen machen keinen Unterschied.

Bei unserer nächsten Therapiesitzung frage ich interessiert nach ihren Ritualen im Ramadan und finde Anhaltspunkte, die einen Beitrag zur Erklärung der Schlafstörungen liefern können. Ashiras Familie, alle sind berufstätig, isst nicht nur nach Sonnenuntergang, sondern auch vor Sonnenaufgang, damit sie genug Kraft für die Arbeit haben. Da in diesem besagten Jahr der Ramadan in die Sommermonate fällt, bedeutet dies, dass um 2:30 Uhr in der Nacht der Wecker läutet, Ashira beim Vorbereiten des Essens hilft und anschließend bis 4 Uhr gegessen wird, danach gehen alle wieder schlafen. Um 6 Uhr läutet der Wecker wieder.

Zuerst galt es nun mit Ashira mögliche Szenen zur Verbesserung ihres Schlafs zu entwickeln. Ich forderte sie auf, mögliche und unmögliche Szenarien zu entwerfen und in psychodramatischen Übungen zu erspüren. Darunter waren, von außen betrachtet, einfache Lösungs-Szenen: Durchschlafen und den Ramadan ignorieren oder alternativ: Durchschlafen und den Ramadan etwas ,,anpassen“. Sie fühlte sich in diesen Szenen nicht wohl, andere wurden kreiert und erprobt, die das Einhalten des Fastens für sie ermöglichten und dennoch ihre Schulnoten nicht gefährdeten. 


\section{6 Überraschungen}

Herr S. kommt heute mit einem Laptop zur Therapiestunde, er lächelt und möchte mir etwas aus seiner Heimat zeigen. Ich bin ob seines Gesichtsausdrucks auf schöne Bilder oder Erinnerungen eingestellt. Herr S. schaltet den Laptop ein, öffnet ein Video und dreht den Computer so zu mir, dass wir beide zusehen können. Zuerst sehe ich eine karge Landschaft, wenige Anhaltspunkte, höre Lärm, es sind Schüsse, dann laufen und fallen Menschen durchs Bild, es ist eine Kriegsszene. Herr S. lächelt und erzählt, dass sein Onkel und sein Bruder ebenfalls in diesem Gebiet ermordet wurden. Nein, sie seien jetzt nicht im Bild, aber es war auch in diesen Bergen. Der Film geht weiter, es ist jetzt nicht mehr viel zu sehen, sondern es sind nur die Schüsse zu hören. Herr S. hat das Video selbst aufgenommen, aber es war verloren gegangen. Es hatte erst gestern, über soziale Medien, wieder den Weg zu ihm gefunden. Herr S. lächelt nach wie vor.

Ich habe damit zu tun, mich selbst zu stabilisieren. Das Video hat mich unvorbereitet getroffen. Ich hatte keine Traumakonfrontation geplant. Ich bedanke mich für das Vertrauen, dass ich das Video sehen durfte und ersuche Herrn S., das Video für heute zu beenden und den Laptop zu schließen. Wir könnten das nächste Mal weiter schauen, wenn Herr S. das möchte. Er schließt den Laptop und erzählt jetzt davon, wie sehr er den Regen mag. Es regnet gerade draußen. Der Regen würde auch der Region, aus der er kommt und die wir gerade im Video gesehen haben, sehr gut tun. Sein Lieblingswetter ist Regen.

Ich habe nur einen kurzen Filmausschnitt in der Nähe der Kampfhandlungen gesehen, bei denen Herr S. dabei war - und doch habe schon ich Mühe, den Schrecken zu verarbeiten und handlungsfähig zu bleiben. Die kurze Erstarrung, die ich selbst gefühlt habe, kennt Herr S. nur zu gut. Sie ist Teil seines alltäglichen Lebens, das weiß ich aus den vorangegangenen Therapiestunden. Ein kurzes Sharing meinerseits bringt Herrn S. wieder mehr ins Hier und Jetzt zurück. Wir bearbeiten die Szene nur soweit es für die Stabilisierung von Herrn S. für diesen Moment notwendig ist. Danach folgen bereits bekannte und immer wieder geübte Stabilisierungsübungen, wie z. B. die Tresorübung oder der Innere Garten (nachzulesen bei Reddemann 2019, S. 66f.). Die weitere Bearbeitung der traumatischen Erfahrungen vereinbaren wir für die nächste Zeit, nach neuerlicher Überprüfung, ob es für Herrn S. passt.

Ich habe Herrn S. ganz bewusst gestoppt, um eine Retraumatisierung zu verhindern und damit während der Therapieeinheit noch genügend Zeit bleibt, die Gegenwart wahrzunehmen, in diesem anderen Land, zu dieser Stunde anzukommen, die traumatischen Erlebnisse auszuhalten und im Prozess integrieren zu können. Noch erlebt Herr S. einen sehr schwankenden Zugang. Entweder berichtet er wie aus weiter Ferne, ohne emotionalen Bezug (Schutz) vom Erlebten oder es gibt einen, wie er es erlebt, unkontrollierten Emotionsausbruch, den er fast nicht aushält. Er fühlt sich ausgeliefert, da er dies nicht steuern kann. Derzeit überwiegt eindeutig die Abspaltung der unaushaltbaren Gefühle. Eine kurze (wiederholte) kognitive Erklärung darüber, was ein Trauma ist, ist sinnvoll. Dies hilft Herrn S., bisher notwendige 
Strategien bei sich zu akzeptieren, wie auch das dosierte Wahrnehmen von Gefühlen besser auszuhalten. Wichtig ist es hier auch, mit ihm immer wieder Strategien zur Regulierung zu erarbeiten. Damit gewinnt er Handlungsmacht. Besonders in kleinen psychodramatischen Szenen können Handlungen erprobt und geübt werden (allerdings nicht zum erlebten Trauma). Sie stärken den Selbstwert und begleiten aus einer passiven Opferrolle. Von unserem therapeutischen Prozess her hätte ich die Erzählung des Traumas jetzt nicht forciert. Allerdings wurde Herr S. am Tag davor durch das aufgetauchte Video selbst überraschend hineingestoßen und hat es mir heute präsentiert. Daher bin ich gefordert, es mit ihm gemeinsam auszuhalten und die Unglaublichkeit von Kampf und Tod zu benennen. Ich wurde Augen- und Gefühls-Zeugin.

Nachdem ich mit Herrn S. gemeinsam die Kriegsszene besprochen und dann mittels Tresorübung für heute sicher aufbewahrt habe, kehren wir ins Hier und Jetzt zurück, zum Regen, und dass Herr S. ohne Schirm spazieren gehen wird, damit er mit seiner Haut die Tropfen spüren kann.

Die Verarbeitung eines Traumas kann umso besser gelingen, je stabiler und sicherer ein Mensch ist und je mehr Resilienz er aufgebaut hat. Das Umfeld von Menschen mit Fluchterfahrung bietet hier oft kontraindizierte Rahmenbedingungen. Umso wichtiger ist es in der Stabilierungsphase, dem Gegenüber eine tragfähige therapeutische Beziehung anzubieten, damit sichere Bindung erlebt werden kann.

\section{Psychodrama-Übungen anpassen}

In Österreich leben wir in einem individualistischen Gesellschaftssystem, während ein Mensch aus einem anderen Land auch aus einem anderen Gesellschaftssystem kommen kann. Bei der Verwendung der wunderbaren psychodramatischen Urelternübung von Stelzig (Stelzig 2013. S. $225 \mathrm{f}$.) ist es wichtig, dies mitzudenken und die Übung ggf. anzupassen.

Bei Gesprächen über Hamdis Eltern wird klar, dass sie ihre Mutter und ihren Vater nicht als Einzelpersonen wahrnimmt, sondern als Einheit. Meine erste Übungsanleitung „Hier die gute Urmutter ... hier der gute Urvater“ wird von Hamdi mit Stirnrunzeln quittiert. Ihr ist nicht klar, was ich jetzt will und was ich meine. Es gilt also die Übung anzupassen. Wir starten mit der ,Urelterneinheit“", um im Laufe der Arbeit doch wieder zu differenzieren. In ihrer Kindheit waren Hamdis leibliche Eltern gut zu ihr, sie haben sich um sie gesorgt, sie haben ihr zu Essen gegeben, sie haben manches Mal mit ihr gespielt und waren immer wieder auch sehr streng. Auf diese Weise können unterschiedliche, förderliche Elternanteile auch im Inneren erarbeitet werden. Das ist auch möglich, wenn die Eltern als Einheit belassen werden. Erst im Laufe der Zeit wird es für Hamdi sichtbar, dass sie sich nach ihrer leiblichen Mutter mehr sehnt als nach ihrem Vater. Wie sich im Laufe des Therapieprozesses herausstellt sind die Beziehung zur Mutter und jene zum Vater doch sehr unterschiedlich. 
Stelzig weist auch darauf hin, wie wichtig es ist, die Botschaften der Ureltern nicht mit denen der leiblichen Eltern zu verwechseln (Stelzig 2013, S. 226). Es hat sich bewährt, den leiblichen Eltern einen eigenen Platz im Raum zu geben, während die Ureltern direkt hinter dem/der KlientIn platziert werden. So fällt ein Auseinanderhalten leichter.

Der Fokus dieser Übung liegt bei Hamdi auf der Entwicklung der inneren „schützenden Ureltern“ bzw. der Entwicklung von inneren stabilisierenden Anteilen. Dies ist für Hamdi leichter, indem sie sie sich als Einheit vorstellt, die innerlich an ihrer Seite steht. Meine Worte auf Deutsch, fasst Hamdi für sich auf arabisch zusammen. Ich nehme die Körpersprache und den Klang der Sprache wahr, ohne die Worte zu verstehen. Nach einem Blickwechsel und dem Nachspüren erfahre ich den Inhalt in einer mir verständlichen Sprache (deutsch oder englisch). Beim darauffolgenden Doppeln kehren wir zu einer für uns beide verständlichen Sprache zurück.

Einzelne besonders bedeutsame Worte wiederhole ich in der Muttersprache der KlientInnen. Allein meine meist holprige Aussprache, Worte, die ich mehrfach wiederholen und erarbeiten muss, bewirken, dass sich die KlientInnen angenommen und wertgeschätzt fühlen und nicht selten ein Lächeln. Das war auch bereits Moreno wichtig, dass Menschen in ihrer Rollenvielfalt wahrgenommen und angesprochen werden, so dass sehr oft auch die Rolle der Lachenden oder des Lächelnden neben sehr belastenden Rollen Platz findet.

Wer Morenos Ehrengrab im Urnenhain der Feuerhalle Simmering besucht, findet dafür auf seinem Grabstein in der Inschrift Bestätigung: „Der Mann, der Freude und Lachen in die Psychiatrie brachte" steht dort zu lesen. Auch Buer befasst sich mit dem Lachen im Psychodrama: „Lachen ist psychologisch gesehen ein spezifischer Atmungsprozess, der darin besteht, dass die Atmung spontan in mehreren unmittelbar hintereinander folgenden Stößen lauthals erfolgt. Das Lachen geht durch den ganzen Körper: es stärkt insbesondere Lunge, Herz, Immunsystem und regt den Stoffwechsel an. Durch die Ausschüttung von Endorphinen setzt Entspannung ein: Es werden Glücksgefühle ausgelöst. [...] Lachen ist ein menschliches Phänomen, das in allen Zeiten und in allen Kulturen vorkommt, wenn es auch unterschiedliche Formen annehmen kann.“ (Buer 2018, S. 348).

\section{Ausblick}

In der psychodramatischen psychotherapeutischen Arbeit mit traumatisierten Menschen mit Fluchterfahrung ist alles, was zur Stabilisierung beiträgt, förderlich. Es geht um Grundlegendes wie einen sicheren Platz zum Schlafen, nicht frieren, etwas $\mathrm{zu}$ essen, aber auch um Themen, die weit darüber hinaus gehen. Nicht selten gibt es berechtigte Ängste vor Übergriffen und Bedrohungen auch im Aufnahmeland. Deshalb ist ein Verhalten von KlientInnen, das v. a. zu Beginn der Therapie Details über deren Identität verschleiert, zu respektieren. Die Unsicherheit über das Bleiberecht erschwert die therapeutische Arbeit, ebenso wie alltägliche alltagsrassistische Begegnungen. Eine ausreichende Stabilität wird, gemeinsam mit der Suche nach 
und Festigung von Ressourcen, prozesshaft erarbeitet. Die Erweiterung des Sozialen Atoms kann unterstützt und begleitet werden. Die Traumatisierung selbst muss nicht zwingend nochmals durchgearbeitet werden, da dies für traumatisierte Menschen extrem belastend sein kann. Flüchtlinge mussten ihre Erlebnisse bereits mehrmals verschiedensten Behörden schildern, da sie als Legitimation für eine Anerkennung als Flüchtling eine Rolle spielen. Es ist an uns, die Spannung auszuhalten, nicht alle Details der Schrecklichkeiten zu kennen und behutsam abzuschätzen, wann es für eine/ $n$ KlientIn heilsam ist, erneut über das Trauma zu sprechen. „Ich musste immer wieder mit Kindern arbeiten, wo Traumatisierungen nur vermutet wurden, [...] Ich bin absolut nicht der Meinung, dass ein nochmaliges Durcherleben der traumatischen Szene zur Heilung nötig ist.“ (Pruckner 2001, S. 127).

Weitere hilfreiche protektive Faktoren reichen von respektvollen Begegnungen auf Augenhöhe, Optimismus ob des Gelingens des Inklusionsprozesses auf allen Seiten über die Erfahrung von Gestaltungsmöglichkeiten bis hin zum Erleben von Selbstwirksamkeit. „Internale Kontrollüberzeugungen und flexible Bewältigungsstrategien ermöglichen eine aktive und handlungsorientierte Haltung gegenüber vielfältigen Herausforderungen und erleichtern die Suche nach und die Anpassung von Lösungen in unterschiedlichen Situationen. Selbstverständlich förderlich wirken auch materielle Ressourcen sowie soziale Unterstützung in Form von Zuwendung oder Wertschätzung und Hilfe bei Orientierung in einer fremden Gesellschaft." (Schnabel und Gottwald 2017, S. 72).

Integration bedeutet, dass Menschen, die ausgegrenzt wurden, wieder dazugehören. Sie werden jedoch weiter als anders (mit Handicap, mit Fluchterfahrung, AusländerIn, transgender etc.) wahrgenommen. Inklusion bedeutet, dass anders sein nichts Besonderes mehr ist. Die Gesellschaft wird so gestaltet, dass Menschen dort einen guten Platz finden können, ohne dass andere Menschen abgewertet oder benachteiligt werden. Jeder Mensch bringt diverse gesellschaftlich wertvolle Beiträge ein. „,Vor dem Hintergrund der Diskussion über die Umsetzung der Menschen- und Grundrechte und vielfältiger Initiativen zur Durchsetzung partikularer Rechte wird ein Konzept benötigt, das es ermöglicht, die Komplexität von Mehrfachidentitäten theoretisch adäquat zu erfassen. In dieser Perspektive wird Inklusion als ein Konzept vorgeschlagen, das eine optimierte, erweiterte oder gar visionäre Vision von Integration bietet.“ (Georgi und Keküllüoglu 2018, S. 44).

Der im Artikel erwähnten Frage nach der Herkunft muss keine böse Absicht zugrunde liegen, dennoch beinhaltet sie bereits eine Ausgrenzung, da sie die Botschaft „nicht von hier" beinhaltet. Obwohl ein so adressierter Mensch Österreich womöglich als sein einziges Zuhause ansieht, bleibt er/sie in den Blicken anderer ,anders“. Die Haltung ,wir von hier“ und ,die anderen von woanders“ kann den Weg zu rassistischen Ressentiments ebnen. „Damit Integration [und Inklusion, Anmerkung der Autorin] funktionieren kann“, müssen wir ,verstehen, dass sie keine Einbahnstraße ist.“ (vgl. dazu Al-Youssef 2021, S. 40).

Funding Open access funding provided by Danube University Krems University for Continuing Education.

Open Access Dieser Artikel wird unter der Creative Commons Namensnennung 4.0 International Lizenz veröffentlicht, welche die Nutzung, Vervielfältigung, Bearbeitung, Verbreitung und Wiedergabe in 
jeglichem Medium und Format erlaubt, sofern Sie den/die ursprünglichen Autor(en) und die Quelle ordnungsgemäß nennen, einen Link zur Creative Commons Lizenz beifügen und angeben, ob Änderungen vorgenommen wurden.

Die in diesem Artikel enthaltenen Bilder und sonstiges Drittmaterial unterliegen ebenfalls der genannten Creative Commons Lizenz, sofern sich aus der Abbildungslegende nichts anderes ergibt. Sofern das betreffende Material nicht unter der genannten Creative Commons Lizenz steht und die betreffende Handlung nicht nach gesetzlichen Vorschriften erlaubt ist, ist für die oben aufgeführten Weiterverwendungen des Materials die Einwilligung des jeweiligen Rechteinhabers einzuholen.

Weitere Details zur Lizenz entnehmen Sie bitte der Lizenzinformation auf http://creativecommons.org/ licenses/by/4.0/deed.de.

\section{Literatur}

Al-Youssef, M. (2021). Sie kommen von hier. Der Standard. 20./21. März 2021. (S. 40). Wien: StandardVerlagsgesellschaft.

Binder, K. (2020). Jedes Leben zählt. In Arbeiter-Samariter-Bund Österreichs (Hrsg.), sam_Report. Nr. 3/ September 2020 (S. 4-5). Wien: Arbeiter-Samariter-Bund Österreichs.

Buer, F. (2018). Das Lachen im Psychodrama. Zeitschrift für Psychodrama und Soziometrie, 17(2), $345-355$.

Dilling, H., \& Freyberger, H. J. (Hrsg.). (2016). Taschenführer zur ICD-10-Klassifikation psychischer Störungen. Bern: Hogrefe.

Dommel, C. (2018). Interreligiöses Lernen. In I. Gogolin, V.B. Georgi, M. Krüger-Potratz, D. Lengye \& K. Sandfuchs (Hrsg.), Handbuch interkulturelle Pädagogik (S. 465-468). Bad Heilbrunn: Julius Klinkhardt.

Georgi, V.B., \& Keküllüoglu, F. (2018). Integration - Inklusion. In I. Gogolin, V. B. Georgi, M. KrügerPotratz, D. Lengye \& K. Sandfuchs (Hrsg.), Handbuch interkulturelle Pädagogik (S. 41-45). Bad Heilbrunn: Julius Klinkhardt.

Hüther, G., Korittko, A., Wolfram, G., \& Besser, L. (2010). Neurobiologische Grundlagen der Herausbildung psychotraumabedingter Symptomatiken. Trauma und Gewalt, 4(1), 18-31.

Imm-Bazlen, U., \& Schmieg, A. (2017). Begleitung von Flüchtlingen mit traumatischen Erfahrungen. Berlin, Heidelberg: Springer.

Judmaier, P., \& Pichlhöfer, C. (2016). Sollte die Welt nicht besser werden? Digitale Medien, Emanzipationspotenzial und Marginalisierung. Stimme. Zeitschrift für Minderheiten. \#98. Social Media in Krisenzeiten. (S. 8-9). Wien: Verein zur Förderung des Zusammenlebens von Minderheiten und Mehrheiten.

Müller, K., \& Schwarz, C. (2020). Fanning the flames of hate: Social media and hate crime. SSRN. https:// doi.org/10.2139/ssrn.3082972.

Ottomeyer, K. (2002). Psychotherapie mit traumatisierten Flüchtlingen - Fallstricke und Handlungsmöglichkeiten. In K. Ottomeyer \& K. Peltzer (Hrsg.), Überleben am Abgrund. Psychotrauma und Menschenrechte (S. 139-170). Klagenfurt/Celovec: Drava.

Ottomeyer, K. (2004). Psychodrama und Trauma. In J. Fürst, K. Ottomeyer \& H. Pruckner (Hrsg.), Psychodrama-Therapie. Ein Handbuch (S. 348-362). Wien: Facultas.

Pries, L., \& Maletzky, M. (2018). Interkulturalität - Multikulturalität - Transkulturalität. In I. Gogolin, V.B. Georgi, M. Krüger-Potratz, D. Lengye \& K. Sandfuchs (Hrsg.), Handbuch interkulturelle Pädagogik (S. 55-60). Bad Heilbrunn: Julius Klinkhardt.

Pruckner, H. (2001). Das Spiel ist der Königsweg der Kinder. München: inScenario.

Pruckner, H. (2018). Monodramatherapie mit traumatisierten Menschen. In S. Kern \& S. Hintermeier (Hrsg.), Psychodrama-Psychotherapie im Einzelsetting. Therapie und Praxis des Monodramas (S. 374-387). Wien: Facultas.

Reddemann, L. (2019). Imagination als heilsame Kraft. Stuttgart: Klett-Cotta.

Reddemann, L., \& Dehner-Rau, C. (2018). Trauma heilen. Ein Übungsbuch für Körper und Seele. Stutgart: Trias Verlag in Georg Thieme Verlag.

Schlichtmeier, V. (2018). Psychotherapeutische Begleitung von Menschen mit Flucht- und Migrationsgeschichten. Zeitschrift für Psychodrama und Soziometrie, 17(2), 281-292. 
Schnabel, K., \& Gottwald, J. M. (2017). Migration und gesellschaftliche Herausforderungen: Hand-lungsorientierte Ansätze zur Förderung der sozialen Teilhabe. Zeitschrift für Psychodrama und Soziometrie, 16(1), 69-80.

Stelzig, M. (2013). Krank ohne Befund. Salzburg: ecowin.

Trinkel, G., \& Kern, S. (2018). Entstehung und Geschichte des Psychodramas. Kühnsdorf/Wien: Skriptum. UNHCR (Hrsg.). (2016). Flucht und Trauma im Kontext Schule. Wien: UNHCR.

Wintersberger, S. (2020). Psychotrauma und die Folgen. Grundlagen der Psychotraumatologie und Traumatherapie/-behandlung. Handout. Wien: zap-wien.

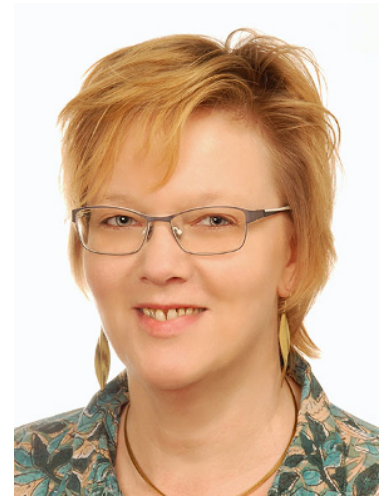

Christine Pichlhöfer Gender- und Kulturorientierte PsychodramaPsychotherapeutin, Lehrbeauftragte für Psychodrama-Psychotherapie im ÖAGG und an der Donau-Universität Krems. Trainerin für Motivational Interviewing. Marginalisierte Gruppen. Seit 1986 im psychosozialen Bereich tätig. Freie Praxis in Wien. 